\title{
- Análise de tendência de mudanças climáticas para a região sul do estado de Minas Gerais no período de 1976 a 2006
}

Brenda Carolina dos Santos Lemos ${ }^{1}$

Leonara Padilha da Silva²

Wezer Lismar Miranda ${ }^{3}$

Leandro Garcia de Abreu4

\section{Resumo}

As alterações climáticas relacionadas ao aquecimento global podem afetar ecossistemas, biomas e ambientes variados. 0 assunto é responsável por amplas discussões no ambiente acadêmico, político e social, com o intuito de desenvolver métodos para reduzir esse processo, o qual é fortemente influenciado pelas ações antrópicas. No entanto, para se constatar tais mudanças é necessária a utilização de critérios científicos, desta forma, objetivou-se com este trabalho analisar a variação da precipitação e das temperaturas mínima, máxima e média do ar nas cidades de Lambari e Lavras, situadas no sul do estado de Minas Gerais. Para tanto, foram coletados dados diários de temperatura mínima, média e máxima do ar ao longo do período de 1976 a 2006, oriundos de Estações Climatológicas Principais instaladas nas cidades citadas. Foram calculadas as temperaturas médias anuais para toda a série por meio da média aritmética dos valores diários para as temperaturas máxima, mínima e média. Para a precipitação pluvial, calculou-se o acumulado anual e, em seguida, foi feita análise de regressão linear para os referidos valores. Os resultados indicaram tendência de aumento da temperatura máxima, média e mínima do ar ao longo do tempo. Quanto à precipitação, não ficou caracterizada uma tendência de acréscimo ou decréscimo em função do baixo valor do coeficiente de determinação.

Palavras-chave: Meteorologia. Temperatura. Mudança Climática.

\section{Introdução}

Nos últimos anos, há uma grande preocupação no ambiente acadêmico e na sociedade em geral a respeito das mudanças climáticas, aquecimento global e seus efeitos para a humanidade. Em trabalhos como os de Bruce (1990) e Berlato, Fontana e Bono (1995), além dessa preocupação, evidenciava-se uma discussão relacionada à possibilidade de mudança climática como conseqüência da emissão de gases de efeito estufa pelas atividades humanas.

De acordo com Blain, Picoli e Lulu (2009), o principal causador da emissão de gases de efeito estufa é a ação antropogênica. 0 processo de maior impacto nessas emissões é a queima de combustíveis fósseis, que tem elevado a concentração de $\mathrm{CO}_{2}$ atmosférico. De fato, o modo de vida da

1 Universidade Vale do Rio Verde, estudante de Engenharia Ambiental. brendacarol@hotmail.com.br. Av. Castelo Branco, 82, Chácara das Rosas, Três Corações (MG), CEP: 37410-000.

2 Universidade Vale do Rio Verde, estudante de Engenharia Ambiental. leonarapadilha@hotmail.com.

3 Universidade Vale do Rio Verde, professor.wmlismar@yahoo.com.br

4 Universidade Vale do Rio Verde, estudante de Engenharia Ambiental. legarcia13@gmail.com. 
sociedade atual é bastante dependente da utilização desses combustíveis e da energia elétrica, por exemplo, para o abastecimento dos variados meios de transporte, para uso em indústrias variadas e na alimentação de equipamentos eletrônicos.

Esse aumento da concentração de $\mathrm{CO}_{2}$ faz com que as mudanças climáticas resultem nas alterações das variáveis meteorológicas, como a precipitação pluvial, temperatura do ar, vento, radiação solar e umidade relativa do ar, ou seja, das variáveis representativas do clima que, ao longo do tempo, geram modificações nos ecossistemas naturais (SANTOS et al., 2010).

Para Silva et al. (2006), investigações sobre a variabilidade e a mudança do clima usam a temperatura média global da superfície para estabelecer o grau e o significado das mudanças do clima durante o último século. No entanto, há que se destacar que em razão de especificidades locais é necessária a averiguação das possíveis tendências de mudanças em microescala.

Para realizar essas averiguações com o devido embasamento técnico-científico é recomendada a utilização de análises estatísticas, comparativamente, entre as variações observadas nos elementos meteorológicos com um período de tempo o mais longo possível. Aplicando método semelhante, Blain et al. (2007), por meio de análise de paralelismo e coincidência de retas geradas por regressão linear simples, indicaram a existência de aumento linear na série de temperatura mínima de Campinas (SP).

Desenvolvendo trabalho semelhante, Altamirano (2010) observou que a região sul do Brasil apresentou uma maior porcentagem de eventos extremos chuvosos em comparação com eventos extremos secos. Ainda, Liebmann et al. (2004), Obregón e Marengo (2007) mostraram tendências significativas de aumento da precipitação total anual na segunda metade do Século XX em praticamente toda a região sul do Brasil.

Por outro lado, muitas tendências de aquecimento observadas nos registros meteorológicos expressam mais os efeitos urbanos locais do que o aquecimento global da atmosfera. 0 surgimento das chamadas "ilhas de calor" devido à urbanização produz o aquecimento da atmosfera que afeta os registros de temperatura, uma vez que a maioria das estações meteorológicas se encontram próximas aos centros urbanos (BACK, 2001).

Assim, com o presente trabalho, objetivou-se avaliar a variação do comportamento dos elementos meteorológicos utilizando as séries anuais de temperaturas máxima, mínima, média e precipitação em duas localidades no sul do estado de Minas Gerais, detectando possíveis tendências de elevação, com intuito de identificar as referidas mudanças climáticas em nível local.

\section{Material e métodos}

Os dados meteorológicos necessários para o presente estudo foram obtidos das estações climatológicas principais pertencentes à rede de observações meteorológicas de superfície do Instituto Nacional de Meteorologia (INMET).

As estações estão situadas nas cidades de Lambari e Lavras, localizadas na região sul do estado de Minas Gerais com as coordenadas geográficas especificadas na Tabela 1 . 
Tabela 1. Coordenadas Geográficas das Estações Climatológicas das quais os dados meteorológicos foram obtidos.

\begin{tabular}{cccc}
\hline Estação & Latitude (S) & Longitude (0) & Altitude (m) \\
\hline Lambari & $21^{\circ} 53^{\prime}$ & $44^{\circ} 39^{\prime}$ & 1.032 \\
Lavras & $21^{\circ} 14^{\prime}$ & $45^{\circ} 00^{\prime}$ & 918 \\
\hline
\end{tabular}

Fonte: Elaborado pelos autores (2016).

Foram utilizados dados diários de precipitação pluvial e temperaturas média, máxima e mínima diárias, correspondentes ao período de 1976 a 2006. Com as informações, procedeu-se à totalização pluviométrica mensal e anual e ao cálculo das médias de temperaturas em escala anual.

Após organizar os dados dessa maneira, plotaram-se gráficos em planilha eletrônica relacionando o período de tempo com os respectivos valores dos elementos meteorológicos analisados.

Para verificação de efetivas tendências de aumento ou decréscimo dos valores observados, utilizou-se para cada variável do ajuste de modelos de regressão linear. Segundo Lima et al. (2012), a regressão linear é um método para se estimar a condicional (valor esperado) de uma variável Y, dados os valores de algumas outras variáveis $X$.

A regressão, em geral, trata da questão de se estimar um valor condicional esperado. Em muitas situações, uma relação linear pode ser válida para sumarizar a associação entre as variáveis Y e X. Assim, podemos apresentar um modelo de regressão linear simples, conforme Equação 1:

$$
Y=\beta_{0}+\beta_{1} X
$$

em que:

$Y$ - variável dependente;

$\beta_{0}$ e $\beta_{1}$ - coeficientes da regressão;

$X$ - variável independente.

Além dessas análises, também foi realizado o Balanço Hídrico Climatológico (BHC) pelo método de Thornthwaite e Mather (1955) para as cidades mencionadas, obtendo a indicação do comportamento da deficiência e do excesso hídrico no período.

Foram utilizadas as Normais Climatológicas (BRASIL, 1992) como referência para comparação do BHC do período de 1961 a 1990 em comparação ao período analisado.

Segundo a classificação climática proposta por Köppen, o clima da região é do tipo Cwa, temperado chuvoso (mesotérmico), com inverno seco e chuvas predominantes no verão, com precipitação total média anual acima de $1.000 \mathrm{~mm}$.

\section{Resultados e discussão}

Os resultados são apresentados nas Figuras de 1 a 5 . As temperaturas máxima, média e mínima do ar, considerando as médias anuais, apresentaram elevação. Resultado semelhante foi observado por Ricce et al. (2009) em estudo realizado com dados meteorológicos observados no município de Londrina (PR) no período de 1961 a 2008. 
Figura 1. Temperaturas máximas anuais para os municípios de Lambari e Lavras (MG)

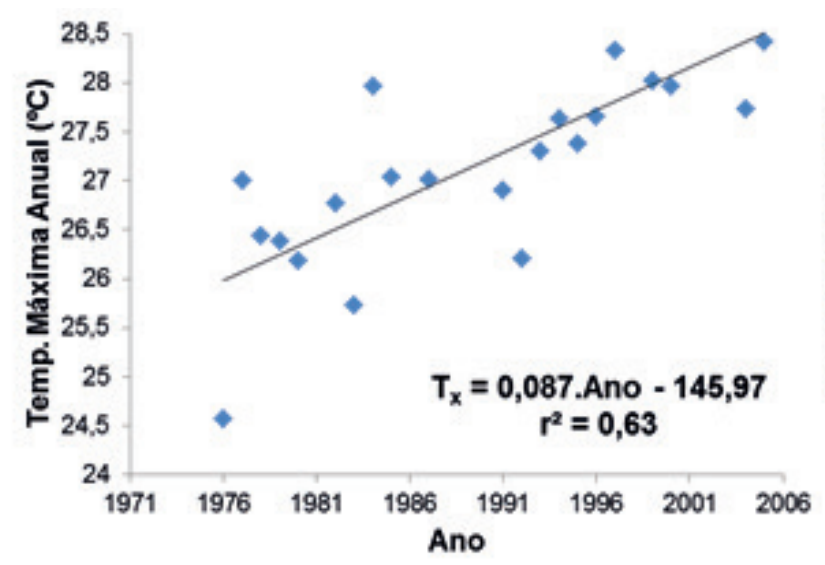

Lambari

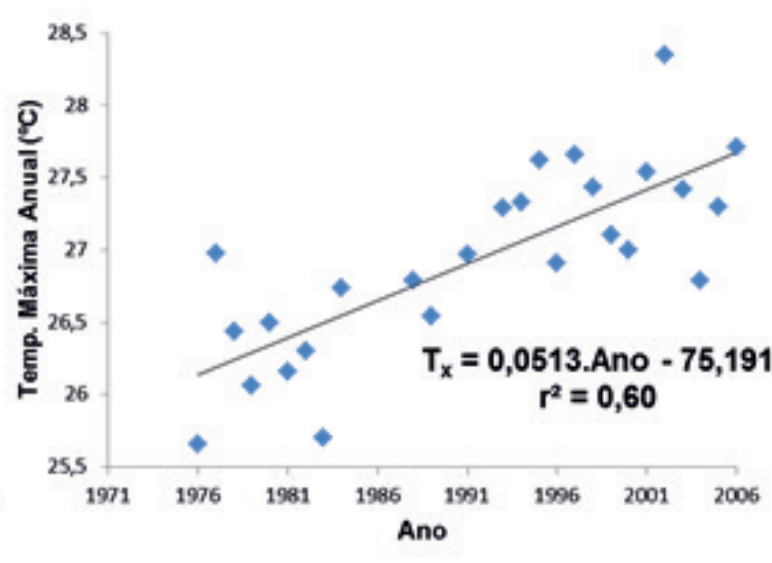

Lavras

Fonte: Elaborado pelos autores (2017).

Figura 2. Temperaturas médias anuais para os municípios de Lambari e Lavras (MG)

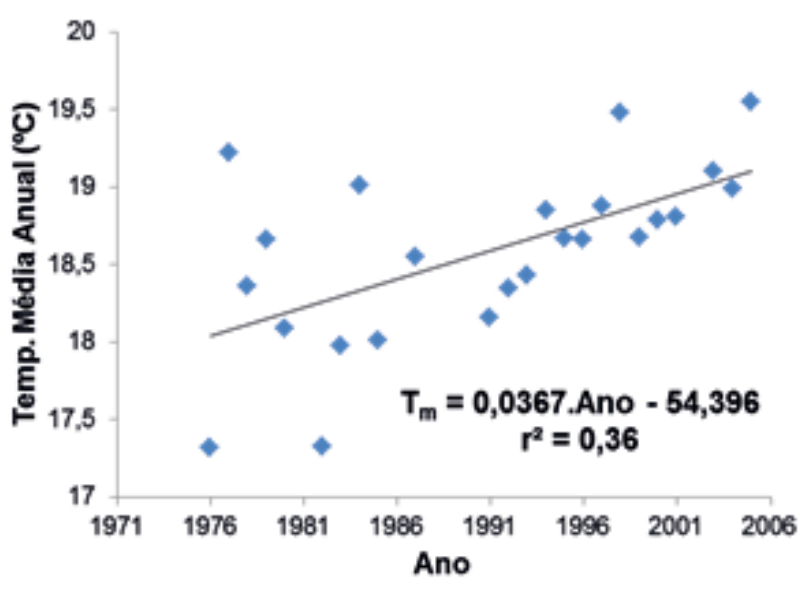

Lambari

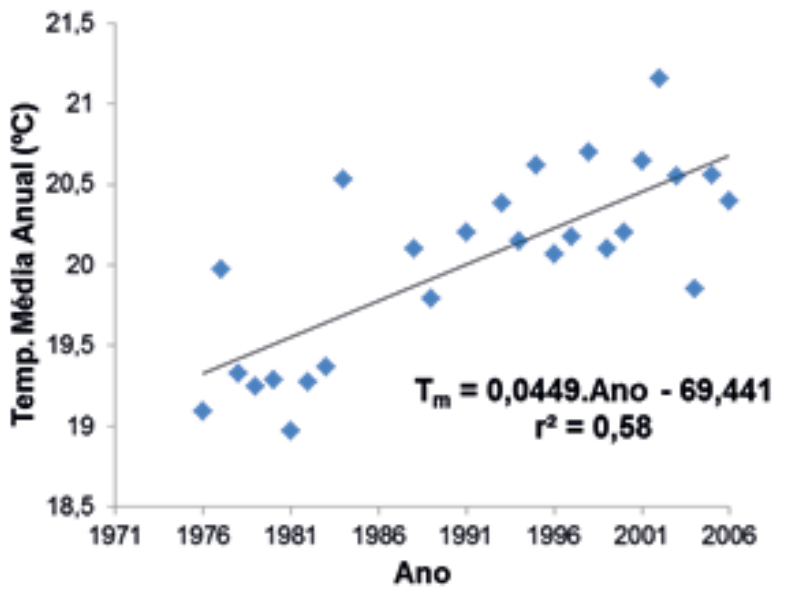

Lavras

Fonte: Elaborado pelos autores (2017). 
Figura 3. Temperaturas mínimas anuais para os municípios de Lambari e Lavras (MG)

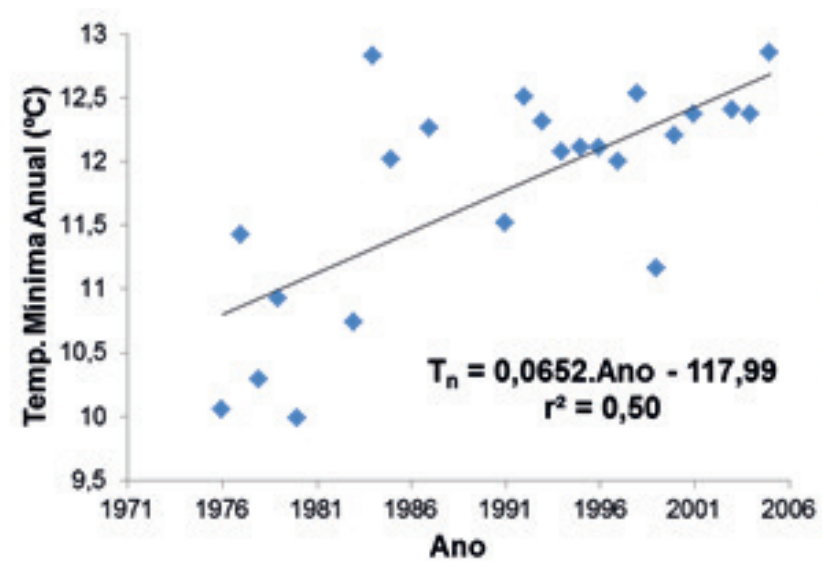

Lambari

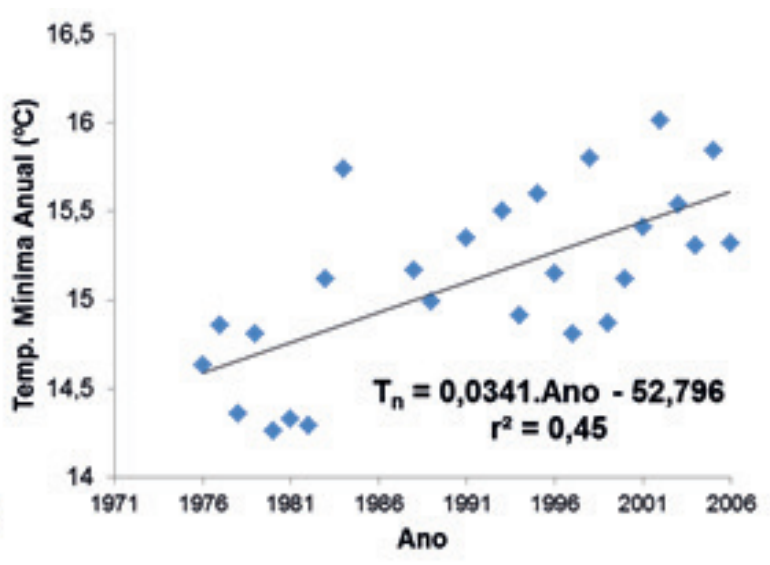

Lavras

Fonte: Elaborado pelos autores (2017).

Nos 30 anos avaliados no presente trabalho, observou-se um aumento de $2,61{ }^{\circ} \mathrm{C}$ e de 1,54 ${ }^{\circ} \mathrm{C}$ na temperatura máxima anual; $1,1{ }^{\circ} \mathrm{C}$ e $1,35^{\circ} \mathrm{C}$ na temperatura média anual; $1,96{ }^{\circ} \mathrm{C}$ e $1,02{ }^{\circ} \mathrm{C}$ na temperatura mínima; respectivamente para os municípios de Lambari e Lavras.

Mello e Alfonsi (1993) e Sentelhas et al. (1994) encontraram valores de aquecimento de até 2 ${ }^{\circ} \mathrm{C}$ em 90 anos na cidade de Campinas (SP), esses valores possivelmente refletem o efeito da urbanização, o que igualmente pode explicar os resultados observados no presente estudo e os relativos baixos valores de $r^{2}$ que variaram de 0,36 a 0,63, indicando que outras variáveis, além do tempo, interferem no processo de elevação da temperatura.

Blain, Picoli e Lulu (2009) observaram tendência de elevação da temperatura mínima anual para as cidades de Campinas, Cordeirópolis e Ribeirão Preto (SP) no período de 1951 a 2006 e destacam que fatores de escala local parecem sobrepor-se a possíveis fatores de escala global, como principais forçantes radioativas no aumento médio dos valores dessa variável meteorológica nas regiões avaliadas.

Observa-se, portanto, a necessidade de avaliar e isolar fenômenos locais relativos à urbanização, por exemplo, antes que investigações de ordem global sejam realizadas em séries históricas de postos meteorológicos.

Quanto à precipitação, Figura 4, os baixos valores de $r^{2}$ não permitem realizar com segurança a inferência de um padrão de comportamento para a variação dos índices pluviométricos. Observando-se as linhas de tendências para ambos os municípios, nota-se que para Lambari a tendência seria de aumento e para Lavras, de redução. 
Figura 4. Precipitação acumulada anualmente para os municípios de Lambari e Lavras (MG)

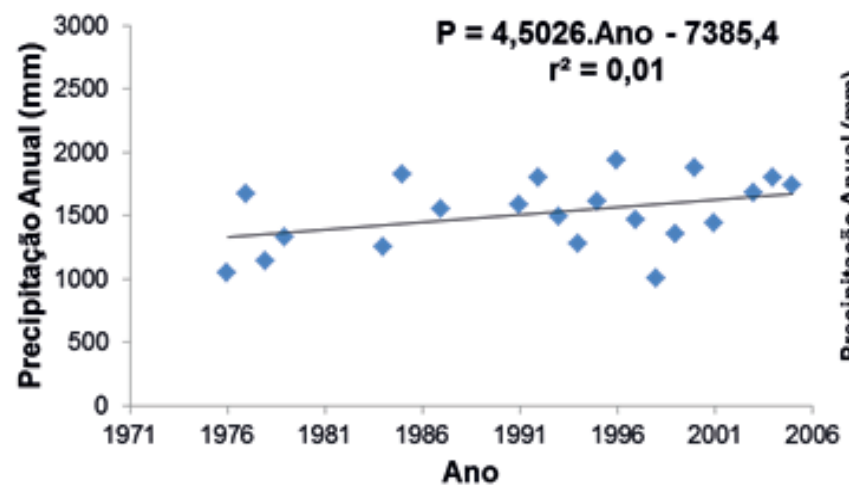

Lambari

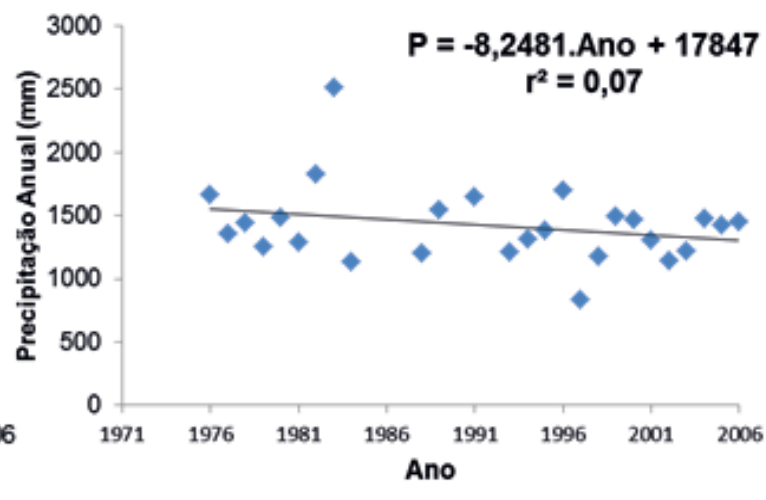

Lavras

Fonte: Elaborado pelos autores (2017).

Esses resultados contrariam o observado por Back (2001) em Urussanga (SC), onde, em análise semelhante, detectou-se tendência de elevação da precipitação pluvial, e o observado por Minuzzi (2010), que não encontrou tendência de mudança no comportamento da precipitação em municípios de Santa Catarina.

Na Figura 5, é apresentado o Balanço Hídrico Climatológico (BHC) observado para o período avaliado, considerando a média entre as duas cidades e o BHC Normal para a região sul do estado de Minas Gerais.

Figura 5. Balanço Hídrico Climatológico para o período avaliado e Normal para a região

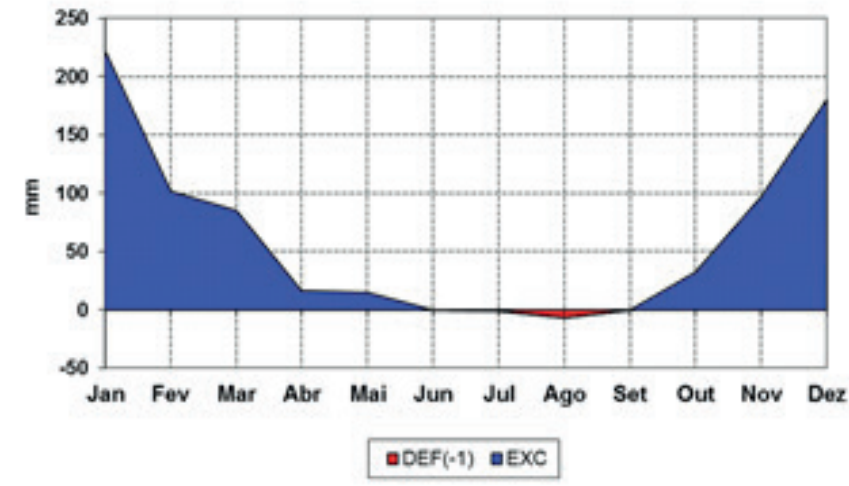

Média Período

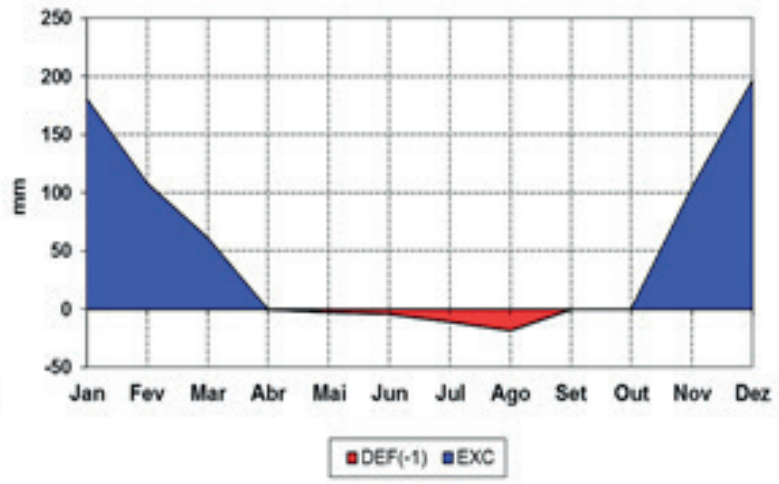

Normal

Fonte: Elaborado pelos autores (2017).

Nesse comparativo é possível observar um prolongamento do período chuvoso até o mês de junho e uma alta concentração da precipitação no mês de janeiro, diferentemente do verificado no período entre 1961 e 1990 indicado pelo gráfico Normal. Desse resultado, depreende-se que há uma tendência de aumento do excedente hídrico e redução da deficiência hídrica. 
Galina (2003), após realizar o processamento do balanço hídrico, analisou a variabilidade e a tendência da deficiência e do excedente hídrico em Ribeirão Preto (SP), verificando aumento na tendência da deficiência hídrica em 49,68 mm e de brusca diminuição na tendência do excedente hídrico em 135,4 mm.

\title{
Conclusões
}

Os valores de temperatura máxima, média e mínima do ar tendem a se manter em elevação ao longo do tempo na região sul do estado de Minas Gerais.

Não foi possível identificar nenhuma tendência para a variação dos índices pluviométricos ao longo do tempo.

A tendência de mudança climática é uma realidade para região sul do estado de Minas Gerais.

\section{Climate change trend analysis for the southern region of Minas Gerais, in the period of 1976 to 2006}

\begin{abstract}
Climate change related to global warming may affect ecosystems, biomes and varied environments. This subject is responsible for extensive discussions in the academic, political and social environment, aiming to develop methods to reduce this process, which is strongly influenced by human actions. In order to investigate these changes, scientific criteria is necessary; therefore, the aim of this study was to analyze the variation in precipitation and minimum, maximum and average air temperature in Lambari and Lavras, cities located in the south of Minas Gerais state. We collected daily data for minimum, average, and maximum air temperature and for precipitation, from 1976 to 2006, provided by Main Climatological Stations operating in the mentioned cities. The annual average temperature for the entire series through the arithmetic average of the daily values were calculated for the maximum, minimum and average temperatures. For the precipitation, the annual accumulation was estimated, followed by linear regression analysis for those values. The results showed a tendency to increase the maximum, average and minimum air temperature over time. For the precipitation, the data did not show a tendency for increasing or decreasing, due to the determination coefficient low value.
\end{abstract}

Keywords: Meteorology. Temperature. Climate Change.

\section{Referências}

ALTAMIRANO, R. J. A. Climatologia dos eventos chuvosos e secos severos, extremos e muito extremos usando o Índice de Precipitação Normalizada (SPI) para as regiões centro-oeste, sudeste e sul do Brasil. 2010. São José dos Campos, Dissertação (Mestrado em Meteorologia) - Instituto Nacional de Pesquisas Espaciais (INPE), 2010.

BACK, A. J. Aplicação de análise estatística para identificação de tendências climáticas. Pesquisa Agropecuária Brasileira, v. 36, n. 5, p. 717-726, 2001. Disponível em: <http://www.scielo.br/scielo. php?pid=S0100-204X2001000500001\&script=sci_abstract\&tIng=pt>. Acesso em: 12 nov. 2016. 
BERLATO, M. A.; FONTANA, D. C.; BONO, L. Tendência temporal da precipitação pluvial anual no Estado do Rio Grande do Sul. Revista Brasileira de Agrometeorologia, v. 3, p. 111-113, 1995. Disponível em: <http://www.scielo.br/scielo.php?script=sci_nlinks\&ref=000115\&pid=S0100204X200100050000100002\&lng=pt>. Acesso em: 02 dez. 2016.

BLAIN, G. C.; PICOLI, M. C. A.; LULU, J. Análises estatísticas das tendências de elevação nas séries anuais de temperatura mínima do ar no estado de São Paulo. Bragantia, v. 68, n. 3, p. 807815, 2009. Disponível em: <http://www.scielo.br/scielo.php?script=sci_abstract\&pid=S000687052009000300030\&lng=es\&nrm=iso\&tlng=pt>. Acesso em: 28 nov. 2016.

BLAIN, G. C.; ROLIM, G.; SENTELHAS, P. C.; LULU, J. Variabilidade temporal da temperatura do ar em Campinas, SP: Análise de tendências e mudanças climáticas. In: CONGRESSO BRASILEIRO DE AGROMETEOROLOGIA, 14., 2007, Aracaju. Anais... Aracaju: SBAgro, 2007. Disponível em: <www.sbagro.org.br/bibliotecavirtual/arquivos/2037.pdf>. Acesso em: 05 dez. 2016.

BRASIL. Ministério da Agricultura, Pecuária e Abastecimento. Normais climatológicas: 1961-1990. Brasília, 1992. 10-24 p.

BRUCE, J. P. The atmosphere of the living planet earth. Genève: World Meteorological Organization, 1990. 31-35 p.

GALINA, M. H. Mudanças climáticas de curto prazo: análise da tendência dos regimes térmicos e hídricos e do balanço hídrico nos municípios de Ribeirão Preto, Campinas e Presidente Prudente (SP), no período de 1969-2001. 2003. Dissertação (mestrado) - Universidade Estadual Paulista, Instituto de Geociências e Ciências Exatas, 2003.

LIEBMANN, B.; VERA, C. S.; CARVALHO, L. M. V.; CAMILONI, I. A.; HOERLING, M. P.; ALLURED, D.; BARROS, V. R.; BÀEZ, J.; BIDEGAIN, M. An Observed Trend in Central South American Precipitation, Journal of Climate, v. 17, p. 4357-4367, 2004. Disponível em: <https://journals.ametsoc.org/ doi/10.1175/3205.1 >. Acesso em: 25 out. 2016.

LIMA, J. G. A.; VIANA, P. C.; WANDERLEY, J. A. C.; MANIÇOSA, R. M.; LIMA, R. M. S. Análise de tendências na temperatura e precipitação em Caicó, Rio Grande do Norte. Agropecuária Científica no Semiárido. v. 8, n. 1, p. 34 -38, 2012. Disponível em: < revistas.ufcg.edu.br/acsa/index.php/ACSA/ article/download/209/pdf>. Acesso em: 10 nov. 2016.

MELLO, M. H. A.; ALFONSI, R. R. A variação térmica secular em Campinas, SP: um exemplo de problemas inerentes ao estudo de séries temporais em climatologia. In: CONGRESSO BRASILEIRO DE AGROMETEOROLOGIA, 7., 1991, Viçosa. Anais... Viçosa: SBAgro, 1993. p. 244-246.

MINUZZI, R. B. Tendências na variabilidade climática de Santa Catarina, Brasil. Revista Brasileira de Engenharia Agrícola e Ambiental, v. 14, n. 12, p. 1288-1293, 2010. Disponível em: www.scielo. br/pdf/rbeaa/v14n12/06.pdf. Acesso em: 12 dez. 2016.

OBREGÓN, G.; MARENGO, J. A. Mudanças climáticas globais e seus efeitos sobre a biodiversidade: caracterização do clima no século XX no Brasil: tendências de chuvas e temperaturas médias e extremas: relatório n 2. São Paulo: CPTEC/INPE, 2007. 23-81 p. 
RICCE, W. S.; CARAMORI, P. H.; MORAIS, H.; SILVA, D. A. B.; ATAÍDE, L. T. Análise de tendências na temperatura e precipitação em Londrina, estado do Paraná. In: CONGRESSO BRASILEIRO DE AGROMETEOROLOGIA, 15., set. 2009. Belo Horizonte. Anais... Belo Horizonte: SBAgro, 2009. p. 1-5. Disponível em: <www.iapar.br/arquivos/Image/agrometeorolgia/.../tendencia_temp_Londrina. pdf>. Acesso em: 15 nov. 2016.

SANTOS, D. N. dos; SILVA, V. P. P. da; SOUSA, F. A. S.; SILVA, R. A. Estudo de alguns cenários climáticos para o nordeste do Brasil. Revista Brasileira de Engenharia Agrícola e Ambiental, v. 14, n. 5, p. 492-500, 2010. Disponível em: <www.scielo.br/scielo.php?script=sci_ arttext\&pid=S1415-43662010000500006 > . Acesso em: 02 dez. 2016.

SENTElHAS, P. C.; CAMARGO, A. P. de; CAMARGO, M. B. P. de; ALFONSI, R. R. Um século de desmatamento: efeitos no regime térmico, pluvial e no balanço hídrico em Campinas, SP. Revista Brasileira de Agrometeorologia, v. 2, p. 99-103, 1994. Disponível em: <www.sbagro.org.br/ bibliotecavirtual/arquivos/44.pdf>. Acesso em: 03 nov. 2016.

SILVA, V. P. R.; SOUSA, F. A. S.; CAVALCANTI, E. P.; SOUZA, E. P.; SILVA, B. B. Teleconnections between sea-surface temperature anomalies and air temperature in northeast Brazil. Journal of Atmospheric and Solar-Terrestrial Physics. v. 68, n. 1, p.781-792, 2006. Disponível em: www.dca. ufcg.edu.br/vapordagua/artigos/Enil_2006_n01.pdf. Acesso em: 10 dez. 2016.

THORNTHWAITE, C. W.; MATHER, J. R. The water balance. Publications in Climatology, New Jersey, Drexel Institute of Technology, 1955. 25-64 p.

\section{Histórico editorial:}

Submetido em: 22/01/2017

Aceito em: 18/07/2017

\section{Como citar:}

$\underline{A B N T}$

LEMOS, B. C. S; SILVA, L. P.; MIRANDA, W. L.; ABREU L. G. Análise de tendência de mudanças climáticas para a região sul do estado de Minas Gerais no período de 1976 a 2006. Revista Agrogeoambiental, Pouso Alegre, v. 10, n. 3, p. 25-33, jul./set. DOI: http://dx.doi.org/10.18406/2316-1817v10n320181163

APA

LEMOS, B. C. S., SILVA, L. P., MIRANDA, W. L.\& ABREU L. G (2018). Análise de tendência de mudanças climáticas para a região sul do estado de Minas Gerais no período de 1976 a 2006.Revista Agrogeoambiental, 10 (3), 25-33. DOI: http://dx.doi.org/10.18406/2316-1817v10n320181163

$\underline{\text { ISO }}$

LEMOS, B. C. S., SILVA, L. P., MIRANDA, W. L. e ABREU L. G. Análise de tendência de mudanças climáticas para a região sul do estado de Minas Gerais no período de 1976 a 2006. Revista Agrogeoambiental, 2018, vol. 10, n. 3, pp. 25-33. Eissn 2316-1817. DOI: http://dx.doi.org/10.18406/2316-1817v10n320181163

\section{VANCOUVER}

Lemos BCS, Silva LP, Miranda WL, Abreu LG. Análise de tendência de mudanças climáticas para a região sul do estado de Minas Gerais no período de 1976 a 2006. Rev agrogeoambiental. 2018. jul./set.; 10(3): 25-33. DOI: http://dx.doi.org/10.18406/2316-1817v10n320181163 


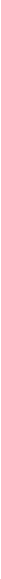

